\title{
Top-side ionosphere response to extreme solar events
}

\author{
A. V. Dmitriev ${ }^{1,2}$, H.-C. Yeh ${ }^{1}$, J.-K. Chao ${ }^{1}$, I. S. Veselovsky ${ }^{2,3}$, S.-Y. Su ${ }^{1}$, and C. C. Fu ${ }^{1}$ \\ ${ }^{1}$ Institute of Space Science, National Central University, Chung-Li, Taiwan \\ ${ }^{2}$ Skobeltsyn Institute of Nuclear Physics Moscow State University, Moscow, Russia \\ ${ }^{3}$ Space Research Institute (IKI) Russian Academy of Sciences, Moscow, Russia
}

Received: 17 August 2005 - Revised: 27 January 2006 - Accepted: 14 Febraury 2006 - Published: 3 July 2006

Part of Special Issue "The 11th International Symposium on Equatorial Aeronomy (ISEA-11), Taipei, May 2005”

\begin{abstract}
Strong X-flares and solar energetic particle (SEP) fluxes are considered as sources of topside ionospheric disturbances observed by the ROCSAT-1/IPEI instrument during the Bastille Day event on 14 July 2000 and the Halloween event on 28 October-4 November 2003. It was found that within a prestorm period in the dayside ionosphere at altitudes of $\sim 600 \mathrm{~km}$ the ion density increased up to $\sim 80 \%$ in response to flare-associated enhancements of the solar X-ray emission. Ionospheric response to the SEP events was revealed both at sunlit and nightside hemispheres, where the ion density increased up to $\sim 40 \%$ and $100 \%$, respectively. We did not find any prominent response of the ion temperature to the X-ray and SEP enhancements. The largest X-ray and SEP impacts were found for the X17 solar flare on 28 October 2003, which was characterized by the most intense fluxes of solar EUV (Tsurutani et al., 2005) and relativistic solar particles (Veselovsky et al., 2004). Solar events on 14 July 2000 and 29 October 2003 demonstrate weaker impacts with respect to their X-ray and SEP intensities. The weakest ionospheric response is observed for the limb X28 solar flare on 4 November 2003. The topside ionosphere response to the extreme solar events is interpreted in terms of the short-duration impact of the solar electromagnetic radiation and the long-lasting impact of the SEP.
\end{abstract}

Keywords. Ionosphere (Solar radiation and cosmic ray effects: Ionospheric disturbances) - Solar physics, astrophysics and astronomy (Energetic particles)

\section{Introduction}

Extreme solar events are characterized by anomalous enhancements of solar hard electromagnetic emission in a frequency range of up to gamma rays and/or of solar energetic

Correspondence to: A. V. Dmitriev

(dalex@jupiter.ss.ncu.edu.tw) particles (SEP), with energies up to hundreds and thousands of MeV. The solar radiation impacts the Earth's ionosphere almost immediately. The impact depends on the spectrum and contents of the radiation (e.g. Mitra, 1974).

The present study regards two outstanding events which occurred in the 23 solar cycle: Bastille Day on 14 July 2000 and the Halloween event on 28 October-4 November 2003. The Bastille Day solar flare on 14 July had a class X5.7/3B such that the GOES-10 detected the peak intensity of 1-min X-ray flux in the 1-8 Angstrom $(\AA)$ range of about $6 \cdot 10^{-4}$ Watts $/ \mathrm{m}^{2}$. The response of the ionospheric total electron contents (TEC) at sunlit hemisphere to that flare is reported to be about 5 TECU (Zhang et al., 2002a; Liu et al., 2004; Tsurutani et al., 2005), which corresponds to an $\sim 7 \%$ increase above the background of $\sim 69$ TECU $\left(1 \mathrm{TECU}=10^{12}\right.$ electrons $/ \mathrm{cm}^{-2}$ ). The Halloween event was accompanied by several extremely strong solar flares. The 28 October flare was class X17/4B and the GOES-10 X-ray peak intensity reached up to $18 \cdot 10^{-4} \mathrm{Watts} / \mathrm{m}^{2}$. Its ionospheric impact, estimated by Tsurutani et al. (2005), and Zhang and Xiao (2005) was, respectively, $\sim 25$ TECU and 17.6 TECU or $\sim 30 \%$ and $\sim 21 \%$ above the background. The X10/3B flare on 29 October (GOES-10 X-ray peak intensity of $\sim 10 \cdot 10^{-4}$ Watts $\left./ \mathrm{m}^{2}\right)$ produced an $\sim 5$ TECU $(\sim 6 \%)$ enhancement (Tsurutani et al., 2005). The largest in the NOAA records X28/3B class flare on 4 November caused an $\sim 5-7$ $(\sim 5-7 \%)$ TECU enhancement (Tsurutani et al., 2005). Note that the X-class of the 4 November 2003 flare was estimated only approximately because of saturation of the GOES X-ray detector in the 1-8 $\AA$ range. Thus, based on the ionospheric D-region response, Thomson et al. (2005) estimated for that flare a peak magnitude of $\mathrm{X} 45 \pm 5$.

Two questions arise from above: 1 . Why did the relatively weaker flare on 28 October causes the largest TEC enhancement? 2. Why did the extraordinary flare on 4 November produce practically the same TEC enhancement as the relatively moderate flares on 14 July and 29 October? Tsurutani

Published by Copernicus GmbH on behalf of the European Geosciences Union. 


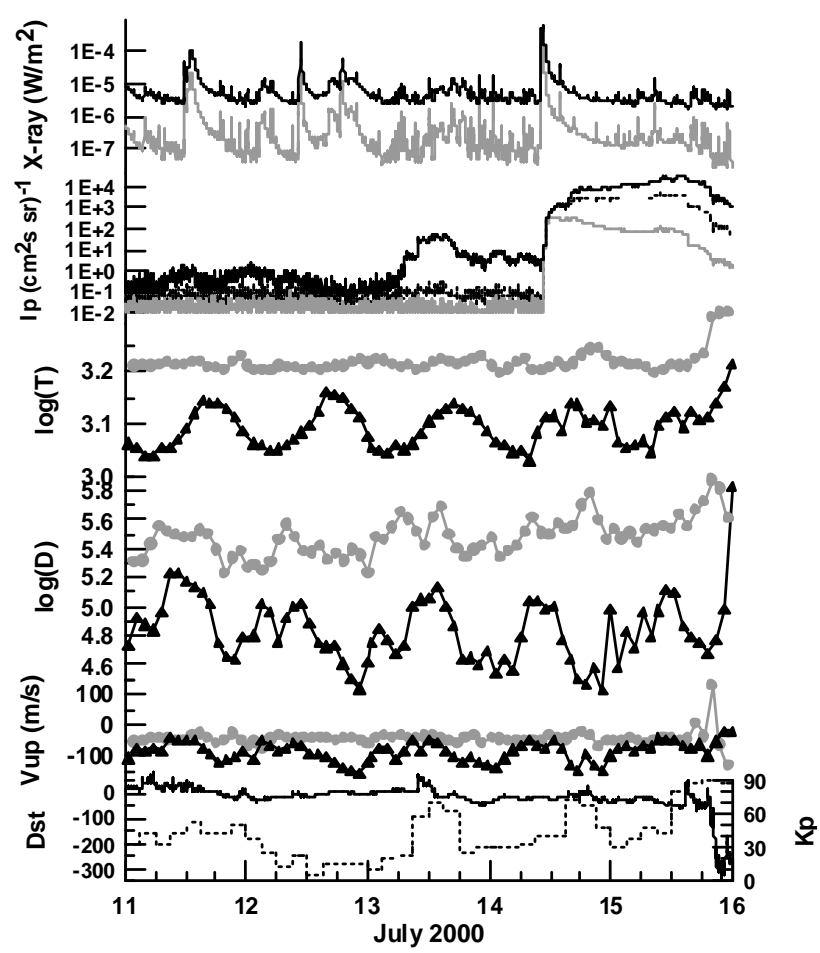

Fig. 1. Solar radiation, ionospheric and geomagnetic conditions preceding the Bastille Day from 11 to 15 July 2000 (from top to bottom): solar X-ray fluxes with wavelength $0.5-4 \AA$ (gray line) and 1-8 $\AA$ (black line); integral fluxes of the interplanetary protons with energies $>5 \mathrm{MeV}$ (black line), $>30 \mathrm{MeV}$ (dashed line) and $>100 \mathrm{MeV}$ (gray line); average temperature $(\mathrm{K})$, density $\left(\mathrm{cm}^{-3}\right)$, and vertical ion drift velocity measured in the top-side ionosphere by the ROCSAT-1/ IPEI during sunlit (gray circles) and nightside passes (black triangles); geomagnetic indices Kp (dashed histogram, right axis) and 1-min Dst (solid line).

et al. (2005) revealed that the EUV spectrum of the 28 October flare is much different from the other flares, namely in the wavelength range $260-340 \AA$ that flare is larger by more than a factor of two. The relatively weak EUV emission of the limb flare on 4 November is due to a strong center-to-limb effect for the EUV (Donnelly, 1976) and, thus, the ionospheric impact of the limb flare should also be smaller (Zhang et al., 2002b).

One more interesting feature reported by Tsurutani et al. (2005) is the remarkable difference between the TEC enhancements and EUV (as well as X-ray) time profiles. Firstly, the TEC peaks are slightly $(\sim 10 \mathrm{~min})$ delayed from the flare peaks. Secondly, the duration of TEC enhancements is lasting several hours, that is much longer than the flare duration. On the other hand, a high correlation was found between the rate of TEC variations and hard X-ray variations observed by Yohkoh in the band of 53-93 keV (Afraimovich et al., 2001; Zhang and Xiao, 2003). In other words, the fast TEC variations are closely related to the solar hard electro-

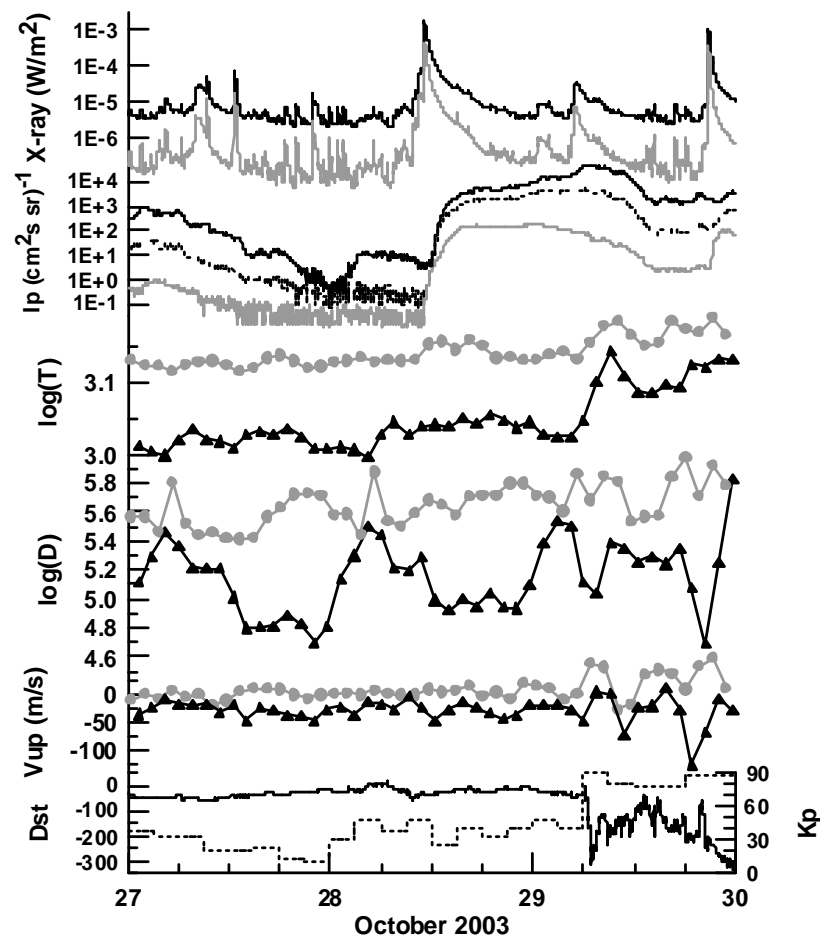

Fig. 2. The same as in Fig. 1 but for the Halloween event on 27-29 October 2003.

magnetic emission while their gradual changes are different. Hence, an additional factor should be considered to explain the difference between the gradual dynamics of the solar emission and ionospheric response. Tsurutani et al. (2005) suggested that the long-lasting TEC variations are controlled significantly by slow (several hours) electron loss via direct recombination at altitudes below $200 \mathrm{~km}$.

Another possible factor affecting the ionosphere is the solar energetic particles (Reid, 1965). In contrast to the fast $(\sim 1 \mathrm{~h})$ flare emission, the SEP enhancements are characterized by gradual variations with characteristic times of several hours. Moreover, the SEP affect high and middle latitudes around the whole globe. Hence, the ionospheric impact of the SEP can be easily distinguished at nightside hemisphere, where effects from the hard electromagnetic emission are minor (e.g. Zhang and Xiao, 2005).

The solar flares on 14 July 2000 and 28 and 29 October 2003 generated extremely intensive fluxes of the relativistic SEP, which ground level enhancements (GLE) were measured by neutron monitors, while the limb flare on 4 November did not produce GLE (Veselovsky et al., 2004). The GLE on 28 October was larger (maximum effect of $\sim 45 \%$ ) than those of the other two GLEs with maximum effects of $\sim 35 \%$. The relativistic SEP fluxes were so intense at $\sim 12$ UT on 28 October that they caused a radiation dose enhancement on board the International Space Station (Panasyuk et al., 2004). 
The topside ionosphere contributes $\sim 25 \%$ to the TEC enhancements (Leonovich et al., 2002). In the present study we analyze the impact of both the solar X-ray emission and SEP to the topside ionosphere at both sunlit and nightside hemispheres during the Bastille Day and Halloween events.

\section{Data}

Solar radiation, ionospheric and geomagnetic conditions during the Bastille Day event on 11-15 July 2000, the Halloween event on 27-29 October 2003 and 2-5 November 2003 are presented in Figs. 1-3, respectively. Data on solar X-rays with wavelengths $0.5-4 \AA$ and $1-8 \AA$ and SEP with energies up to $>100 \mathrm{MeV}$ measured by GOES-10 satellite were acquired from the SPIDR database (http://spidr.ngdc. noaa.gov/spidr/). Note that the fluxes of protons with energies of tens of $\mathrm{MeV}$ observed at geosynchronous orbit are very close to that observed in the interplanetary medium (Cohen et al., 2001). Geomagnetic conditions are characterized by the Kp index and 1-min Dst index, which are provided by Kyoto WDC (http://swdcdb.kugi.kyoto-u.ac.jp).

Topside ionospheric conditions are observed by a nearEarth satellite ROCSAT-1 (Chang et al., 1999). The satellite has a circular Sun-synchronous day-night orbit with $\sim 600$ $\mathrm{km}$ height and $35^{\circ}$ inclination, which permits reaching up to $48^{\circ}$ of the geomagnetic latitude. The ionospheric Plasma and Electrodynamics Instrument (IPEI) on board the ROCSAT-1 consists of an ion trap, a pair of ion drift meters and a retarding potential analyzer (Yeh et al., 1999). The IPEI provides experimental information about such main ionospheric plasma characteristics as ion density, temperature, composition and a vector of drift velocity, with high (up to $10^{-3} \mathrm{~s}$ ) temporal resolution.

In the present study we use IPEI data on the ion density, temperature and vertical drift velocity with 1-s resolution. The ion density corresponds to a local balance among the ionization, recombination and transport processes in a quasisteady-state ionosphere. The temperature variation reflects an intensity of energy change. The vertical velocity of ionospheric plasma reveals the dynamical processes.

For statistical analysis the 1-s data were averaged within $\sim 45$-min intervals, during which the ROCSAT-1 was located at sunlit or at nightside hemispheres. Note that the averaging procedure mixes the ionospheric conditions at low and middle (up to $48^{\circ}$ ) geomagnetic latitudes. Figures 1-3 show the time profiles of the averaged ionospheric parameters for the considered time intervals. We separate variations of the dayside and nightside ionospheric conditions, which have substantially different dynamics. One can see prominent diurnal variations of the ionospheric parameters, especially at the nightside hemisphere. This is due to the variation of the longitudinal and latitudinal location of the satellite, namely the South Atlantic Anomaly (SAA) significantly affects the ion

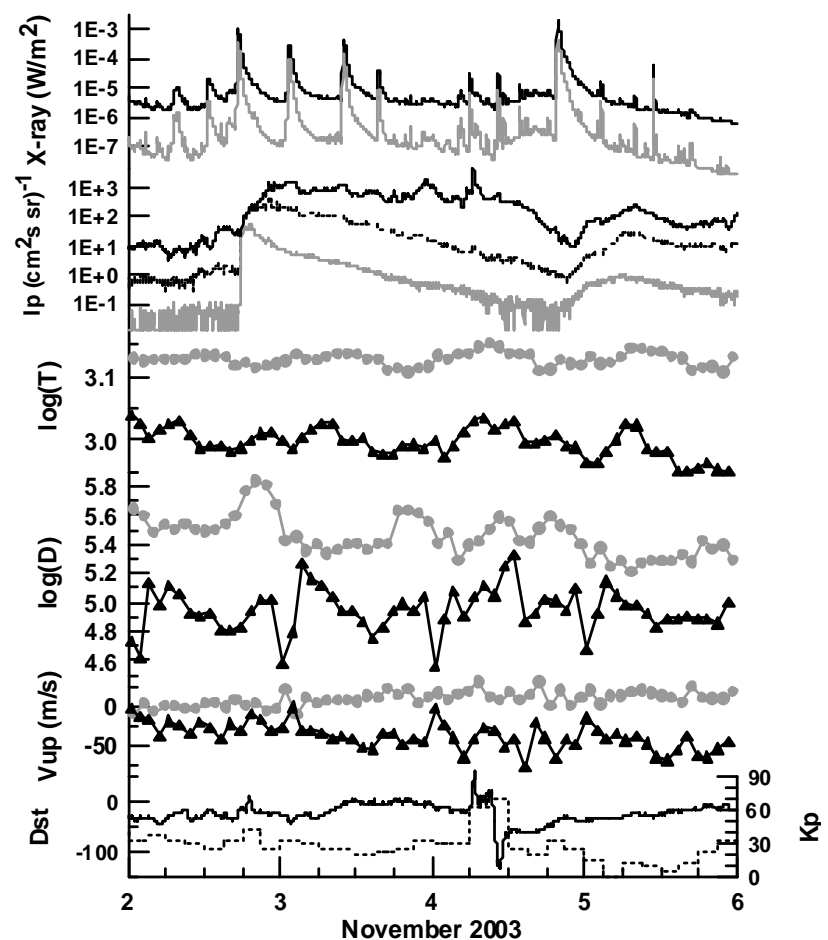

Fig. 3. The same as in Fig. 1 but for time interval 2-5 November 2003.

density and velocity. The ion temperature is higher when the satellite passes higher geomagnetic latitudes.

The $\sim 45$-min averaging permits studying the ionospheric response to solar radiation enhancements with a characteristic duration of tens of minutes. The solar radiation effects can be hidden by the diurnal variations and also by geomagnetic disturbances. Geomagnetic storms perturb strongly the entire ionosphere (e.g. Basu et al., 2001; Lin et al., 2005; Mannucci et al., 2005; Yizengaw et al., 2005), such that the ion temperature, density and velocity variations become irregular and grow to hundreds of percents in comparison with the prestorm conditions. Accurate determination of the ionospheric background conditions is required for the identification and numerical estimation of the solar radiation impact.

\section{Background problem}

A problem of the background conditions is solved in two steps. Firstly, we select geomagnetically quiet days. We also take into account on the appearance and magnitude of X-ray flares and SEP fluxes at those days. Secondly, we determine and subtract the diurnal variations at the quiet days from the ionospheric variations accompanied with the solar radiation enhancements.

The geomagnetic disturbances can be revealed easily as increases in the $\mathrm{Kp}$ index and/or as large negative Dst variations. An interval on 11-13 July 2000 preceding the Bastille 


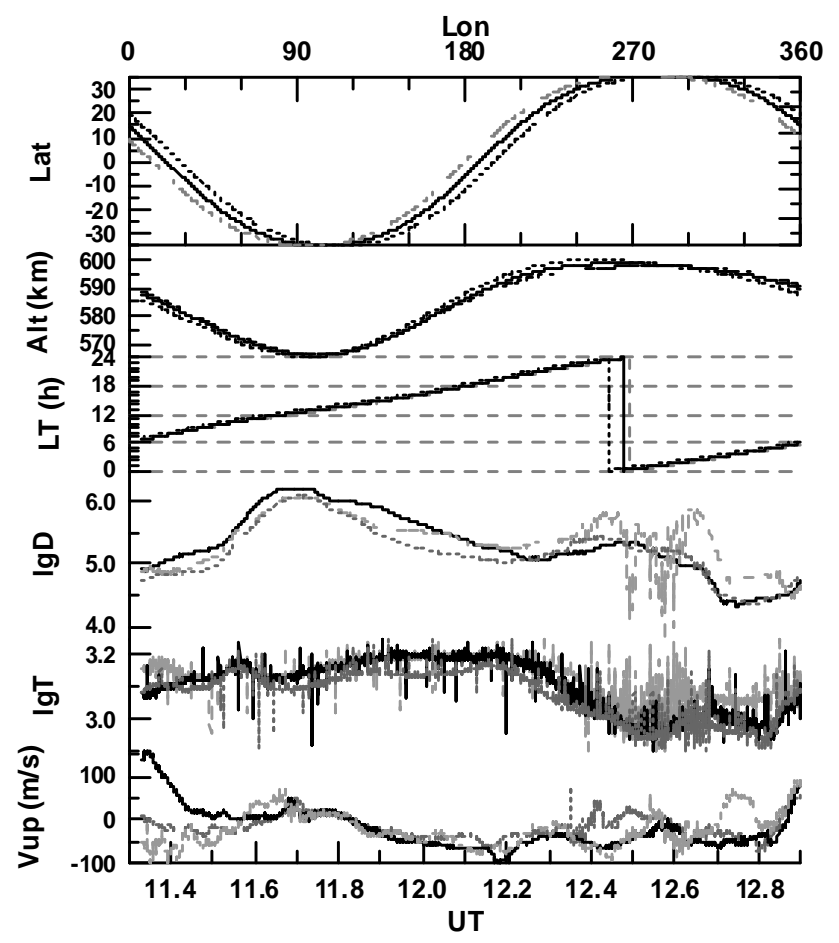

Fig. 4. An adjustment of three ROCSAT-1 orbits on 27 October (gray dotted lines, 3-min time delay), 28 (solid lines, no delay) and 29 (gray dashed lines, -4 min time delay). The panels indicate (from top to bottom): the satellite geographic location, altitude, local time, measured ion density and temperature in log scale, and vertical ion drift velocity. The ionospheric conditions for different passes are much different, despite their spatial location is very close.

Day is presented in Fig. 1. Several solar X-ray enhancements and magnetic storms with $\mathrm{Kp}$ up to 7 accompany that interval. A geomagnetically quiet day is 12 July, but it is slightly disturbed by the X2 solar flare at $\sim 10: 30$ UT. The Bastille event was initiated by a X5.7/3B solar flare, which occurred at 10:24 UT on 14 July in an active region 9077 , located very close to the center of the Sun's visible disk. The flare caused very effective acceleration of the SEP up to relativistic energies. Integral fluxes of the SEP with energies $>100 \mathrm{MeV}$ exceede 300 particles $/\left(\mathrm{cm}^{2} \mathrm{~s}\right.$ sr). There were no prominent SEP enhancements on 12 July. Hence we can neglect the effect from the X2 flare and accept 12 July as a day with background ionospheric conditions.

During the Halloween event we consider separately two time intervals: $27-29$ October and 2-6 November 2003. As one can see in Fig. 2, 27 October is a pretty quiet day. There were no strong X-ray flares, SEP enhancements or geomagnetic disturbances on that day. Hence, ionospheric conditions on 27 October can be considered as a background for perturbations on 28-29 October, which were caused by extremely intensive X-ray flares, SEP enhancements and a strong geomagnetic storm. The latter one began at $\sim 06$ UT, on 29 October and disturbed the ionosphere dramatically. During the

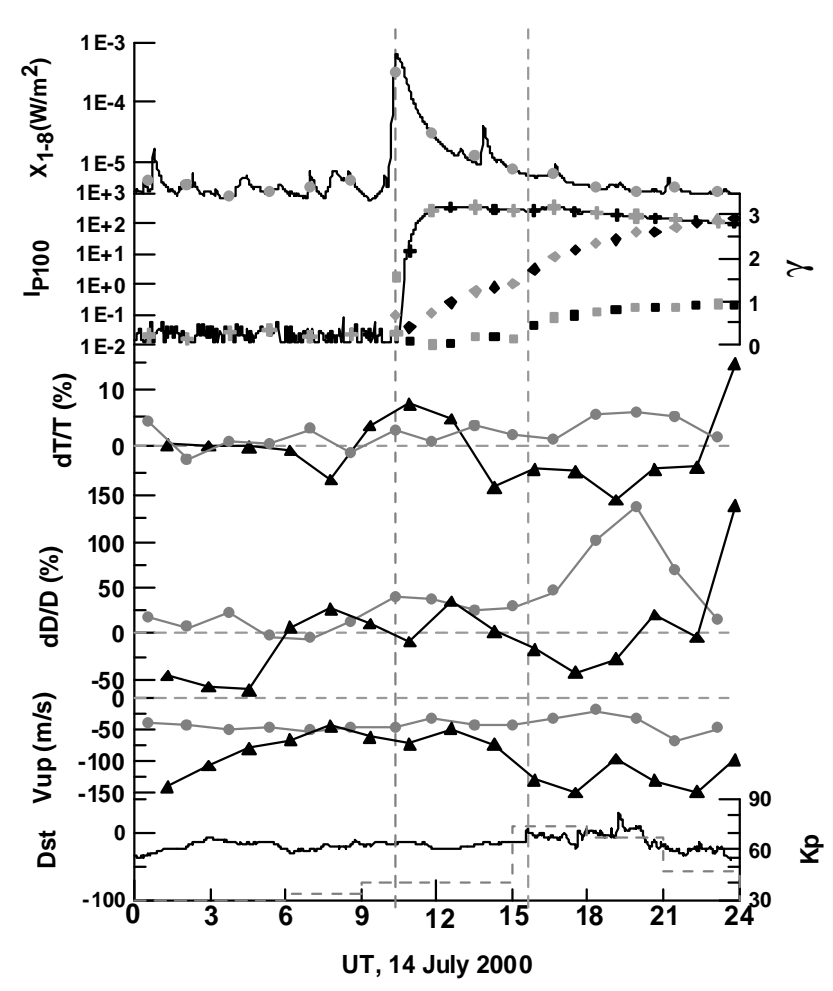

Fig. 5. Topside ionosphere response to the solar event on 14 July 2000 (from top to bottom): solar 1-8 $\AA$ X-ray emission and its $\sim 45-$ min averages (circles); integral flux $\mathrm{I}_{P 100}$ of the SEP with energies $>100 \mathrm{MeV}$ (solid line), its $\sim 45$-min averages (crosses), and average spectral indices $\gamma_{60}$ (diamonds) and $\gamma_{10}$ (squares); relative changes of the ion temperature $d T / T$ and density $d D / D$; vertical ion drift velocity $V_{u p}$; geomagnetic indices $\mathrm{Kp}$ (dashed histogram, right axis) and 1-min Dst (solid line). Gray and black symbols indicate $\sim 45$-min averages calculated, respectively, for sunlit and nightside passes of the ROCSAT-1. Vertical dashed lines depict the solar flare at 10:20 UT and geomagnetic storm onset at 15:35 UT.

storm the topside ionosphere temperature, density and fluctuations of the vertical velocity increased substantially.

The time interval on 2-4 November 2003 (see Fig. 3) is accompanied by several intense $\mathrm{X}$-ray flares and moderate SEP enhancements. Moreover, there is a negative temporal trend in the topside ionospheric temperature and density. This is due to a poststorm enhancement of the equatorial ionization anomaly (Lin et al., 2005). The 5 November day looks relatively quiet. There are no storms or X-ray flares on that day, and the SEP flux becomes weak as well. Thus, we selected that day as background for the ionospheric perturbations on 4 November which were caused by strong geomagnetic storms and by an extremely intense X-ray flare.

In order to subtract the background accurately we adjust the ROCSAT-1 orbits at the quiet days to mostly close orbits at the disturbed days. Figure 4 demonstrates an example of the adjustment of three ROCSAT- 1 orbits on 27, 28 


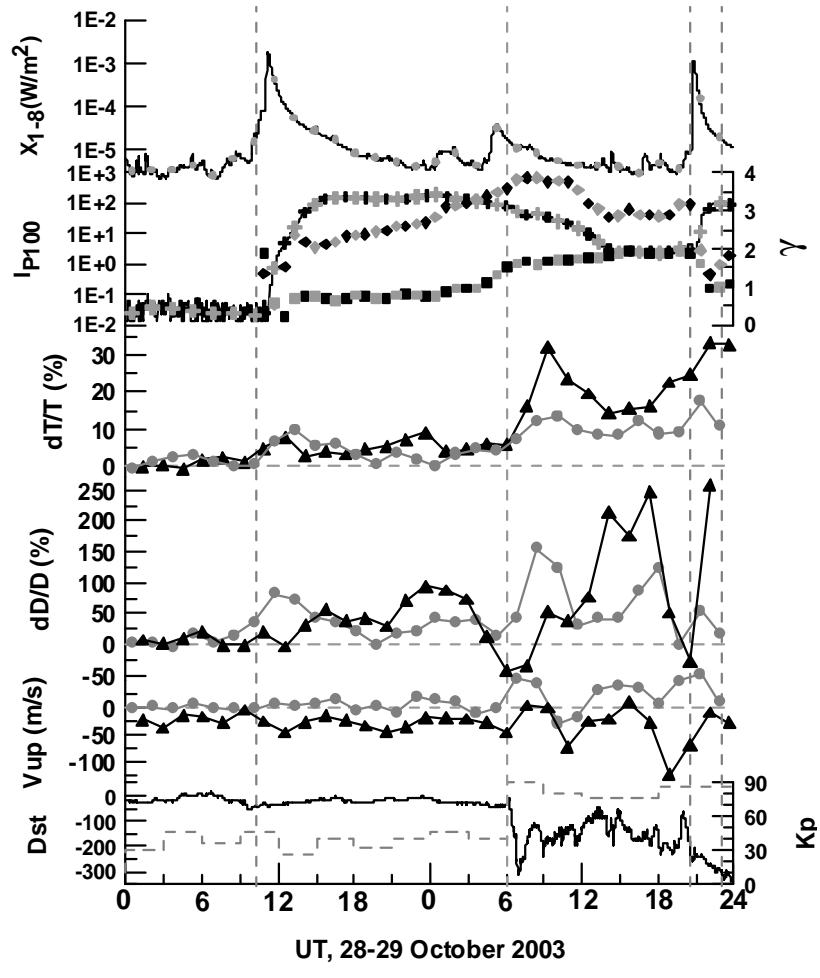

Fig. 6. The same as in Fig. 5 but for the Halloween event on 28-29 October, 2003. Vertical dashed lines depict solar flares at 10:05 UT, on 28 October and at $\sim 21: 30 \mathrm{UT}$, on 29 October, and the geomagnetic storm sudden commencement at $\sim 06: 10 \mathrm{UT}$, on 29 October.

and 29 October 2003. The passes on 27 and 29 October are shifted on 3 min and -4 min, respectively, to obtain the best coincidence with the pass at 11:20-12:55 UT on 28 October. One can see that the latitudes, altitudes and local times of the adjusted orbits are very close. Moreover, the ion density and temperature profiles are pretty similar, except fot the nighttime storm-associated features.

By this way we adjust all the orbits on 14 July with 12 July 2000 (background day), on 28 and 29 October with 27 October 2003 (background day), and on 4 November with 5 November (background day). Subtracting the background at quiet days, we suppress the diurnal variations. The residual time profiles bring us information about the solar radiation impact to the topside ionosphere.

\section{Analysis}

From the residual data we calculate the relative changes in the ion density $d D / D$ and temperature $d T / T$ as $\left(P_{\text {dist }}{ }^{-}\right.$ $\left.P_{b g}\right) / P_{b g}$, where $P_{\text {dist }}$ and $P_{b g}$ are $\sim 45$-min averaged values at disturbed and quiet (background) days, respectively. Figures 5-7 show the results of the subtraction. Note that we distinguish dayside and nightside passes in order to separate

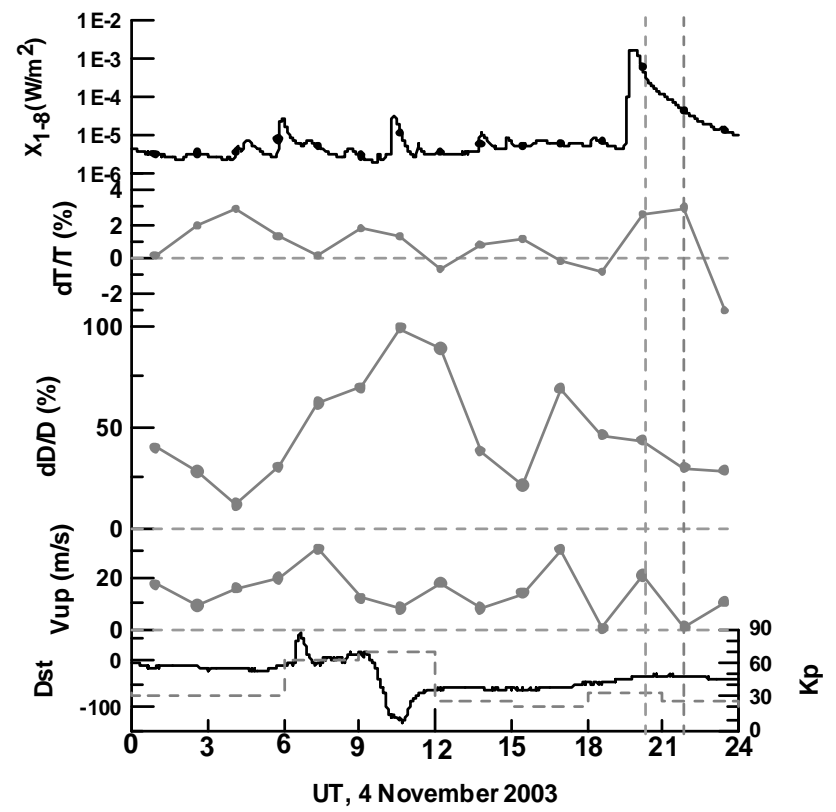

Fig. 7. Strongly perturbed topside ionosphere at sunlit hemisphere before the X28 solar flare at 21:00 UT on 4 November 2003 (from top to bottom): solar X-ray emission with wavelengths 1-8 $\AA$; relative changes of the ion temperature $d T / T$ and density $d D / D$; vertical ion drift velocity Vup; geomagnetic indices $\mathrm{Kp}$ (dashed histogram, right axis) and 1-min Dst (solid line). Circles indicate values of the parameters, which are averaged within daytime passes of the ROCSAT-1. Vertical dashed lines depict the interval around the solar flare time.

the X-ray effect at the sunlit hemisphere from the SEP radiation effects at both sunlit and nightside hemispheres.

For a qualitative analysis we use $\sim 45$-min averages of the $1-8 \AA$ solar X-ray flux, corresponding to the ROCSAT1 passes at sunlit hemisphere. For both dayside and nightside passes we calculate $\sim 45$-min averages of the $>100 \mathrm{MeV}$ SEP flux. A spectrum of the SEP is characterized by average spectral indices $\gamma_{60}$ and $\gamma_{10}$ in the energy ranges of $>60 \mathrm{MeV}$ and $>10 \mathrm{MeV}$, respectively. We assume here a power shape of the spectrum, such that $\gamma_{E 1}=\log \left(\mathrm{I}_{E 1} / \mathrm{I}_{E 2}\right) / \log (\mathrm{E} 2 / \mathrm{E} 1)$, where $\mathrm{I}_{E 1}$ and $\mathrm{I}_{E 2}$ are $\sim 45$-min averaged integral fluxes of the SEP with energies $>E 1$ and $>E 2$, respectively. For the energy range $>60(>10) \mathrm{MeV}$ we take $\mathrm{E} 1=60(10) \mathrm{MeV}$ and $\mathrm{E} 2=100(60) \mathrm{MeV}$.

Figure 5 demonstrates the ionospheric response to the X5.7 flare on 14 July 2000. Before the flare onset at $\sim 10: 10$ UT the ionospheric conditions are close to the background ( $d T / T \sim 0$ and $d D / D \sim 0)$, except for the nightside region at 00:00-05:00 UT. The latter is probably due to a redundant effect of the geomagnetic storm on 11 July (see Fig. 1). At $\sim 10: 10-10: 30$ UT the ROCSAT-1 observes the ion density increase up to $40 \%$ at the sunlit hemisphere. There is no prominent change in the ion density on 


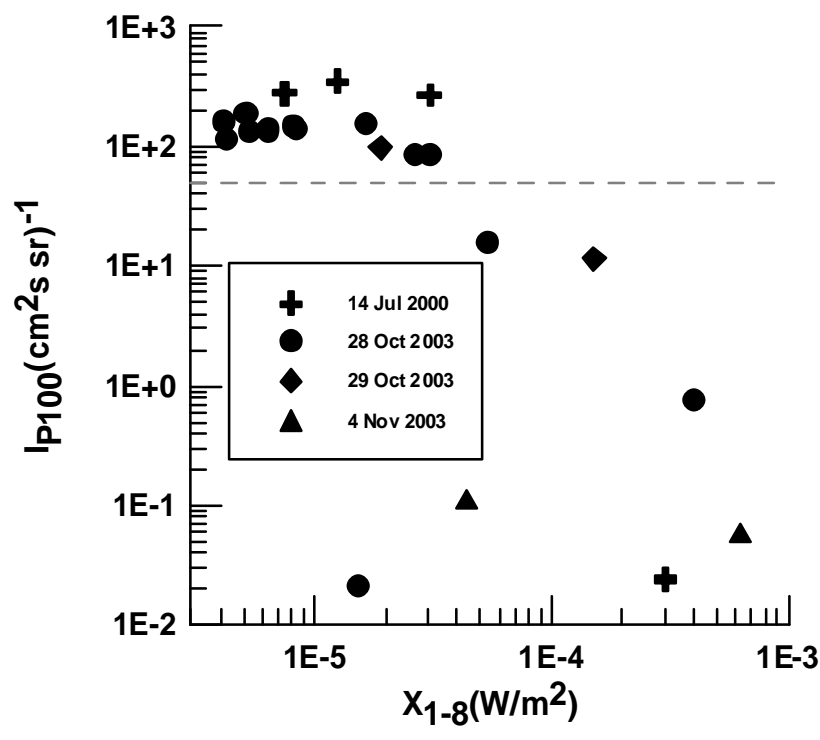

Fig. 8. Scatter plot of the average $>100 \mathrm{MeV}$ SEP fluxes versus average solar 1-8 $\AA$ X-ray fluxes corresponding to the ROCSAT-1 passes at sunlit hemisphere during the considered solar events (depicted by vertical dashed lines in Figs. 5-7). Horizontal dashed line restricts a lower threshold for the predominant SEP effects.

the nightside. At that time the temperature increases only slightly $(<10 \%)$ at sunlit hemisphere.

During the following dayside pass at 11:20-12:10 UT and nightside pass at 12:15-13:00 UT the relativistic SEP fluxes significantly enhance, while the $\mathrm{X}$-ray flux decreases dramatically. The ion plasma density increases both at the sunlit and nightside hemispheres. At that time the SEP spectral index $\gamma_{10}$ is small and the $\gamma_{60}$ gradually increases, which indicates the decrease in the relativistic SEP fluxes. After the geomagnetic storm commencement at 15:35 UT the ionospheric dynamics changes dramatically and the solar radiation effect is hidden by the high amplitude storm-time ionospheric perturbations.

A much more prominent solar radiation impact to the ionosphere is revealed on 28 October (Fig. 6). The dayside ion density and temperature vary well with the X17 flare dynamics and the peak density is estimated to be $>80 \%$. From $\sim$ 12:00 UT to 24:00 UT, on 28 October, the spectral indices of the enhanced SEP fluxes change slowly. The index $\gamma_{10}$ stays about 0.7 and $\gamma_{60}$ increases gradually from 2 to 2.5 . Such spectral dynamics indicates that the SEP fluxes are effectively accelerated to very high energies by the interplanetary shock ahead of an extremely fast CME (Dmitriev et al., 2005). At that time the ion temperature and density increase at both sunlit and nightside hemispheres. Since 00:00 UT, on 29 October, the SEP spectrum is softening with the CME approaching the Earth. The high-energy SEP flux is decreasing and the ionospheric density and temperature are dropping.
Since 06:00 UT the ionospheric conditions are controlled by the great geomagnetic storm. At the end of 29 October, during dayside passes of the ROCSAT-1 at 21:00-21:45 UT and at 22:35-23:15 UT, the ionospheric temperature and ion density demonstrate a prominent positive change, which is accompanied by the X10 solar flare. The SEP effect to the ionosphere is difficult to identify at that time because of significant storm-time ionospheric variations, especially at nightside hemisphere.

It is important to note that the solar radiation does not influence the vertical ion drift velocity. As can be clearly seen in Figs. 5-7, the drift velocity at the sunlit hemisphere does not demonstrate any prominent response to the sudden increase of solar X-ray emission. During the SEP enhancements the pattern of velocity variations is similar to the undisturbed conditions. However, the variations increase dramatically during the geomagnetic storms.

A positive response of the sunlit ionospheric density and temperature to an extremely intensive X28 solar flare at 19:50-20:35 UT and at 21:30-22:15 on 4 November 2003 is shown in Fig. 7. In the present case the solar flare impacts the ionosphere, which has already been perturbed by the strong geomagnetic storm at $\sim 06: 00-1200$ UT. It is rather difficult to estimate the relative contributions of the solar flare and poststorm effects to the ionospheric perturbations. In the first approach we attribute the ion density and temperature variations to the $\mathrm{X}$-ray flare effect only. However, the actual ionospheric response to the flare could be smaller than the observed variation.

The radiation conditions affecting the dayside ionosphere during the extreme solar events are summarized on a scatter plot of the SEP fluxes versus solar X-ray emission (Fig. 8). Two regions can be distinguished from the scatter plot. The first one corresponds to very intense SEP fluxes of more than $50\left(\mathrm{~cm}^{2} \mathrm{~s} \mathrm{sr}\right)^{-1}$ with relatively weak X-ray fluxes. Hence, the SEP effects can be assumed to be predominant in that region. The second region is located below the threshold of $50\left(\mathrm{~cm}^{2} \mathrm{~s} \mathrm{sr}\right)^{-1}$, where the X-rays are very intense. Thus, in that region the solar X-ray effect prevails. Note that this is not a strict separation, namely both the X-ray and SEP effects contribute significantly to the points characterized by the $\mathrm{X}$ ray flux above $4^{*} 10^{-5} \mathrm{~W} / \mathrm{m}^{2}$ and the SEP flux of more than $10\left(\mathrm{~cm}^{2} \mathrm{~s} \mathrm{sr}\right)^{-1}$. However, in the first approach we neglect the latter effect.

Figure 9 shows the topside ionosphere response to the Xray fluxes which corresponds to the second region. Despite a wide scattering of the data points, one can see a positive proportionality between the logarithm of the 1-8 $\AA$ flux and the relative changes in the ion density. The response of ion temperature is very much scattered and it does not exceed $10 \%$. The most prominent temperature variation of $\sim 17 \%$ on 29 October is substantially contributed to by storm-time geomagnetic disturbances. Hence it is difficult to conclude about the temperature dependence on the X-ray emission. 


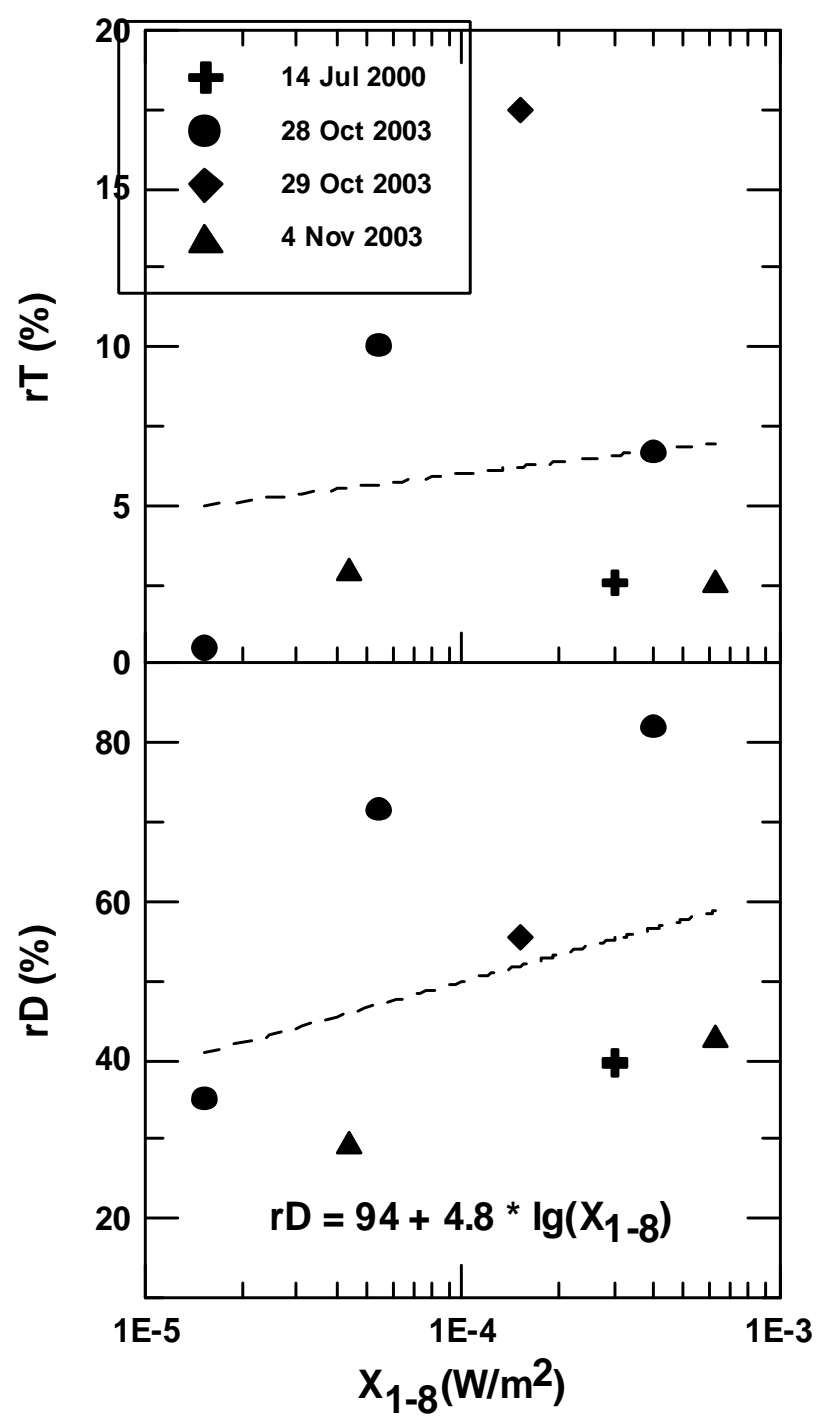

Fig. 9. Relative change in the topside ionospheric temperature (upper panel) and ion density (lower panel) versus solar 1-8 $\mathrm{X}$-ray fluxes.

Ionospheric effects of the SEP at sunlit and nighside hemispheres are summarized in the scatter plots presented in Fig. 10. Statistically, the ionospheric density increases with the SEP fluxes. The SEP effect on the dayside is about two times smaller than that of the X-ray effect (Fig. 9). On the other hand, the response of ion density is much larger at night, such that the density increases up to $\sim 100 \%$ on 28 October. It is rather difficult to fit the points in Fig. 10b because of very wide scattering. For the SEP event on 28 October 2003 one can see that the relative change in the ionospheric density increases proportionally to the logarithm of the proton flux. However, the Bastille Day event breaks that pattern. The ion temperature response of $\sim 10 \%$ is scattered very widely, so we cannot find any relationship between the temperature and the $>100 \mathrm{MeV}$ SEP fluxes.

\section{Discussion and summary}

From the analyses of the Bastille Day and Halloween events we have found the topside ionosphere responses to both solar X-ray and SEP enhancements. The ion density increases by tens of percents with increasing X-ray and SEP intensity. The solar radiation enhancements are accompanied with enhanced ion temperature (up to $10 \%$ ). However, we did not find a direct proportionality between the ionospheric temperature and the X-ray or SEP intensity. Note that the ionospheric response to the solar radiation is much weaker than the storm-time ionospheric perturbations.

We should emphasize that the solar X-rays and SEP expose different spatial regions of the ionosphere. The solar electromagnetic emission mainly influences the subsolar region (Zhang and Xiao, 2005), while the SEP affects the ionosphere at high and middle latitudes. Note that in classical dipole approach (e.g. Reid, 1965) the solar protons with energy $\sim 100 \mathrm{MeV}$ are unable to penetrate directly to the ROCSAT-1 orbit, due to a high cutoff threshold of $>3 \mathrm{GeV}$ at geomagnetic latitudes $<48^{\circ}$. However, for considering the SEP penetration the dipole approach is too rough. Leske et al. (2001) reported that the protons with energy $\sim 30 \mathrm{MeV}$ penetrate directly to geomagnetic latitudes of $\sim 64^{\circ}$ (instead of $\sim 80^{\circ}$ in the dipole approach) during quiet geomagnetic conditions and even to lower latitudes during geomagnetic storms. Apparently the SEP, with energies of about hundreds of $\mathrm{MeV}$ should penetrate to lower latitudes.

Perhaps the observed ionospheric response to the SEP is due to ionization at middle and high latitudes. Moreover, the SEP penetration boundary is located at lower latitudes in the nighttime (Ivanova et al., 1985), which could partially explain the larger response of the ionospheric density to the SEP at the nightside hemisphere. As we have found the SEP impact at night is comparable to the $\mathrm{X}$-ray impact at the sunlit hemisphere.

The wide spreading of the ionospheric response indicated in Fig. 10 can be partially explained by various maximum geomagnetic latitudes, which are passed by the ROCSAT-1. A stronger response corresponds to higher latitudes. Moreover, for some sunlit passes, the SEP and X-ray effects are overlapped. Hence, the sunlit ionospheric response should be fitted as a two-parametric function of the X-ray and SEP fluxes with an additional correction on the orbit location. Because more data are required for such a fitting, in the present study we consider the X-ray and SEP effects separately. However, as one can see in Fig. 8, the separation is sometime ambiguous. Hence, the numerical dependencies presented in Figs. 9 and 10 should be considered as a first approach.

Quantitative comparison of the topside ionosphere responses to the X-ray solar flares (see Fig. 9) has revealed that the largest increase in the ion density occurred on 28 October. The flares on 29 October and 14 July are less intensive and their ionospheric impacts are also weaker, accordingly. The ionospheric response to the X28 limb flare on 4 November 

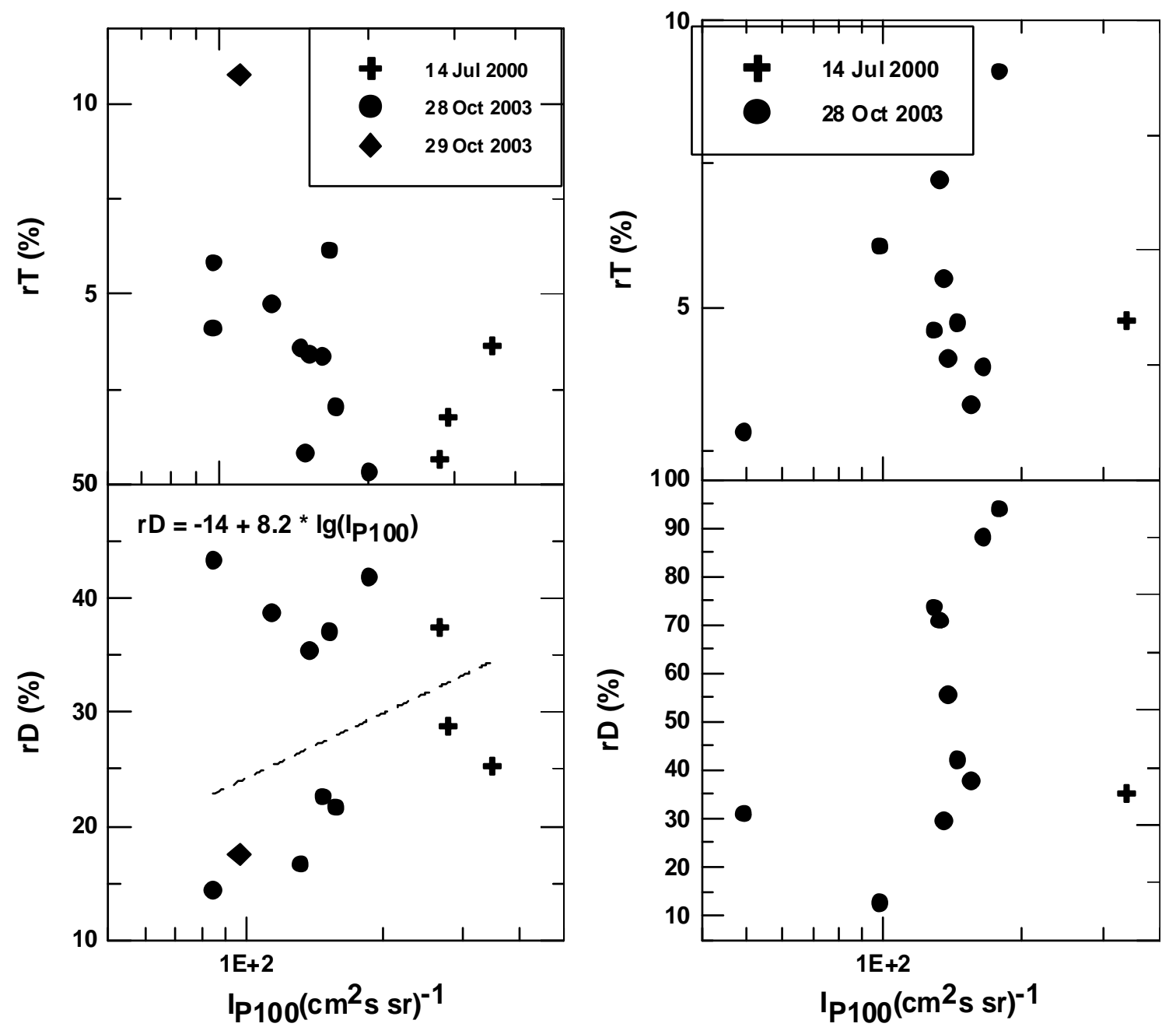

(a)

(b)

Fig. 10. Relative change of the topside ionospheric temperature (upper panels) and ion density (lower panels) versus $>100 \mathrm{MeV}$ SEP fluxes at the sunlit (a) and the nightside (b) hemispheres.

is the weakest. This is in good agreement with the previous studies of the TEC response to the X-ray flares.

The strongest SEP impact to the ionosphere is also observed on 28 October. Ionospheric responses to the SEP on 14 July and 29 October are weaker, though the most intensive SEP fluxes are detected on 14 July. This inconsistency can be explained by the difference in the SEP spectra. Indeed, it has been reported that the highest maximum effect of the GLE (up to $45 \%$ ) was detected on 28 October. This is because more intensive fluxes of the relativistic solar particles were observed than those observed on 14 July and 29 October. The difference between the relativistic parts of SEP spectra can also explain significant scattering of the ionospheric response to the $>100 \mathrm{MeV}$ SEP fluxes (see Fig. 10). We can therefore suggest the SEP impact as an additional factor which contributes to the long-lasting TEC enhancements reported by
Tsurutani et al. (2005), especially at middle and high latitudes.

Finally, we can interpret the different ionospheric responses to the extreme solar events. The X-ray flares have different impacts on the topside ionosphere, as well as on the TEC, depending on the different EUV spectra of the flares as suggested by Tsurutani et al. (2005). The largest ion density and TEC enhancement on 28 October are caused by the most intense EUV emission from the X17 central solar flare on that day. The largest SEP impact on the ionosphere is also revealed for the solar event on 28 October which was accompanied by very intense fluxes of high energy SEP with hard spectrum. This event is characterized by the most intense fluxes of the relativistic solar particles. The high-energy SEP fluxes can contribute also to the unusually long-lasting TEC enhancements observed after the extreme solar events. 
Acknowledgements. The authors thank NOAA Space Physics Interactive Data Resource for providing data from the GOES satellites and Kyoto World Data Center for Geomagnetism for providing the geomagnetic indices. This work was supported by grants NSC93-2811-M-008-024 and NSC94-2811-M-008-039. ISV acknowledges the partial support by grants INTAS 03-51-6206, RFBR 04-02-16736.

Topical Editor M. Pinnock thanks E. L. Afraimovich and another referee for their help in evaluating this paper.

\section{References}

Afraimovich, E. L., Altyntsev, A. T., Kosogorov, E. A., Larina, N. S., and Leonovich, L. A.: Ionospheric effects of the solar flares of September 23, 1998 and July 29, 1999 as deduced from global GPS network data, J. Atmos. Sol. Terr. Phys., 63(17), 18411849, 2001.

Basu, S., Basu, S., Valladares, C. E., Yeh, H.-C., Su, S.-Y., MacKenzie, E., Sultan, P. J., et al.: Ionospheric effects of major magnetic storms during the International Space Weather Period of September and October 1999: GPS observations, VHF/UHF scintillations, and in situ density structures at middle and equatorial latitudes, J. Geophys. Res., 106(A12), 30 389-30 413, 2001.

Chang, Y.-S., Chiang, W.-L., Yiang, S.-J., Holt, B. J., Lippincott, C. R., and Hsieh, K. C.: System Architecture of the IPEI Payload Onboard ROCSAT-1, TAO, Supplementary Issue, 7-18, 1999.

Cohen, C. M. S., Mewaldt, R. A., Cummings, A. C., Leske, R. A., Stone, E. C., Slocum, P. L., Wiedenbeck, M. E., et al.: Forecasting the arrival of shock-accelerated solar energetic particles at Earth, J. Geophys. Res., 106, 20 979-20 984, 2001.

Dmitriev, A. V., Chao, J.-K., Suvorova, A. V., Ackerson, K., Ishisaka, K., Kasaba, Y., Kojima, H., and Matsumoto, H.: Indirect estimation of the solar wind conditions in 29-31 October 2003, J. Geophys. Res., 110, A09S02, doi:10.1029/2004JA010806, 2005.

Donnelly, R. F.: Empirical models of solar flare X-ray and EUV emissions for use in studying their $\mathrm{E}$ and $\mathrm{F}$ region effects, J. Geophys. Res., 81, 4745-4753, 1976.

Ivanova, T. A., Kuznetsov S. N., Sosnovets, E. N., and Tverskaya, L. V.: Dinamics of low-latitude boundary for penetration solar low-energy protons in the magnetosphere, Geomag. Aeron. (in Russian), 25(1), 7-12, 1985.

Leonovich, L. A., Afraimovich, E. L., Romanova, E. B., and Taschilin, A. V.: Estimating the contribution from different ionospheric regions to the TEC response to the solar flares using data from the international GPS network, Ann. Geophys., 20, 19351941, 2002.

Leske, R. A., Mewaldt, R. A., Stone, E. C., and von Rosenvinge, T. T.: Observations of geomagnetic cutoff variations during solar energetic particle events and implications for the radiation environment at the Space Station, J. Geophys. Res., 106(A12), 30 011-30 022, 2001.

Lin, C. H., Richmond, A. D., Liu, J. Y., Yeh, H. C., Paxton, L. J., Lu, G., Tsai, H. F., and Su, S.-Y.: Large-scale variations of the low-latitude ionosphere during the October-November 2003 superstorm: Observational results, J. Geophys. Res., 110, A09S28, doi:10.1029/2004JA010900, 2005.
Liu, J. Y., Lin, C. H., Tsai, H. F., and Liou, Y. A.: Ionospheric solar flare effects monitored by the ground-based GPS receivers: Theory and observation, J. Geophys. Res., 109, A01307, doi:10.1029/2003JA009931, 2004.

Mannucci, A. J., Tsurutani, B. T., Iijima, B. A., Komjathy, A., Saito, A., Gonzalez, W. D., Guarnieri, F. L., et al.: Dayside global ionospheric response to the major interplanetary events of October 29-30, 2003 "Halloween Storms", Geophys. Res. Lett., 32(12), L12S02, doi:10.1029/2004GL021467, 2005.

Mitra, A. P.: Ionospheric effect of solar flares, D. Reidel, Norwell, Mass., 1974.

Panasyuk, M. I., Kuznetsov, S. N., Lazutin, L. L., Avdyushin, S. I., Alexeev, I. I., Ammosov, P. P., Antonova, A. E., et al.: Magnetic Storms in October 2003, Cosmic Res., 42(5), 489-534, 2004.

Reid, G. C.: Solar Cosmic Rays and the Ionosphere, in: Physics of the Earth's upper atmosphere, edited by: Hines, C. O., Paghis, I., Hartz, T. R., and Fejer, J. A., Prentice-Hall, Inc., Englewood Cliffs, N. J., 1965

Thomson, N. R., Rodger, C. J., and Clilverd, M. A.: Large solar flares and their ionospheric $D$ region enhancements, J. Geophys. Res., 110, A06306, doi:10.1029/2005JA011008, 2005.

Tsurutani, B. T., Judge, D. L., Guarnieri, F. L., Gangopadhyay, P., Jones, A. R., Nuttall, J., Zambon, G. A., et al.: The October 28, 2003 extreme EUV solar flare and resultant extreme ionospheric effects: Comparison to other Halloween events and the Bastille Day event, Geophys. Res. Lett., 32(3), L03S09, doi:10.1029/2004GL021475, 2005.

Veselovsky, I. S., Panasyuk, M. I., Avdyushin, S. I., Bazilevskaya, G. A., Belov, A. V., Bogachev, S. A., Bogod, V. M., et al.: Solar and heliospheric phenomena in October-November 2003: causes and effects, Cosmic Res., 42(5), 435-488, 2004.

Yeh, H. C., Su, S. Y., Yeh, Y. C., Wu, J. M., Heelis, R. A., and Holt, B. J.: Scientific Mission of the IPEI Payload Onboard ROCSAT1, TAO, Supplementary Issue, 19-42, 1999.

Yizengaw, E., Moldwin, M. B., Dyson, P. L., and Immel, T. J.: Southern Hemisphere ionosphere and plasmasphere response to the interplanetary shock event of 29-31 October 2003, J. Geophys. Res., 110, A09S30, doi:10.1029/2004JA010920, 2005.

Zhang, D. H., Xiao, Z., Igarashi, K., and Ma, G. Y.: GPS-derived ionospheric TEC response to a solar flare that occurred on 14 July 2000, Radio Sci., 37(5), 1086, doi:10.1029/2001RS002542, 2002a.

Zhang, D. H., Xiao, Z., and Chang, Q.: The correlation of flare's location on solar disc and the sudden increase of total electron content, Chin. Sci. Bull., 47(1), 82-85, 2002b.

Zhang, D. H. and Xiao, Z.: Study of the ionospheric total electron content response to the great flare on 15 April 2001 using the International GPS Service network for the whole sunlit hemisphere, J. Geophys. Res., 108(A8), 1330, doi:10.1029/2002JA009822, 2003.

Zhang, D. H. and Xiao, Z.: Study of ionospheric response to the 4B flare on 28 October 2003 using International GPS Service network data, J. Geophys. Res., 110, A03307, doi:10.1029/2004JA010738, 2005. 